\title{
Atención de urgencias en Cartagena, Colombia
}

\author{
Emergency care service in the city Cartagena Colombia
}

Elías Alberto Bedoya Marrugo ${ }^{1}$

\section{Resumen}

Objetivo. Analizar las características de la atención de urgencias en la ciudad de Cartagena, tomando como parámetro inicial los factores revisados de manera documental en diferentes fuentes, de las cuales sobresale la encuesta nacional de salud pública versión 2007 y datos obtenidos a nivel internacional. Métodos. Estudio descriptivo consistente en una búsqueda documental y estadística de cifras y conceptos relacionados de diferentes autores y organizaciones relacionadas con la atención y observación de la atención sanitaria, con especial énfasis en la atención de urgencia, tomando como guía los estudios relevantes de la encuesta nacional de salud y la observación de los servicios hospitalarios de emergencia (EHS) en medio oriente, europa y España. Resultados. La atención de urgencias en Cartagena es uno de los servicios críticos en el cual un $86 \%$ de la atención obedece a motivos de consulta no obstétricos y donde $55 \%$ de los solicitantes son mujeres, mientras el $45 \%$ restante son hombres y donde el 31\% de los pacientes presentan incomodidades al momento de ser atendidos.

Palabras claves: Urgencias, hospital, triage.

\begin{abstract}
Objective. analyze the characteristics of emergency care in the city of Cartagena, on the initial parameter revised documentary sources in different factors, of which overselling the National Public Health Survey 2007 version and data obtained from a internationally. Method. a descriptive study consistent documentary and statistical search of figures and concepts of different authors and organizations related to the care and observation of health care, with special emphasis on emergency care, guided by relevant studies of the survey national Health and observation of hospital emergency services (EHS) in middle East, Europe and Spain. Result. The emergency care in Cartagena is one of the critical services where $86 \%$ of care due to reasons not obstetric consultation and where $55 \%$ of applicants are women, while the remaining $45 \%$ are men and where $31 \%$ of patients have discomfort when being served.
\end{abstract}

Keywords: Emergency, hospital,triage.

1. Coordinador de investigación, Fundación universitaria Tecnológico Comfenalco, Facultad de ingeniería, Programa de Tecnología en seguridad e higiene ocupacional. 


\section{Introducción}

Conocida la urgencia como la alteración de la integridad física y/o mental de una persona, causada por un trauma o por una enfermedad de cualquier etiología que genere una demanda de atención médica inmediata y efectiva tendiente a disminuir los riesgos de invalidez y muerte donde se requiere que se ejecuten todas las acciones realizadas a una persona con patología de urgencia y que tiendan a estabilizarla en sus signos vitales, realizar un diagnóstico de impresión y definirle el destino inmediato, tomando como base el nivel de atención y el grado de complejidad de la entidad que realiza la atención inicial de urgencia, al tener los principios éticos y las normas que determinan las acciones y el comportamiento del personal de salud (1).

El conocimiento de las principales causas de atención a urgencias médicas en un área de salud no solo permite al médico estar preparado para combatirlas, sino que le proporciona información sobre que enfermedades necesita realizar una mayor prevención, con el objetivos de evitar hechos fatales que pongan en riesgo la calidad de vida del paciente, o den al traste con la vida del mismo, debido al protagonismo que ejerce actualmente la atención primaria en los servicios de urgencia (2). El aumento de la longevidad ha obligado a su mayor interés en los problemas de urgencia de los ancianos.

Hasta ahora no se había demostrado la superioridad de un método aislado de planificación, dirección, equipamiento o funcionamiento de un servicio de urgencias que considere la contradictoria finalidad del tratamiento del paciente de paso, del enfermo grave, o del lesionado (3). Los hospitales de las comunidades deben responder a las necesidades comunitarias al proporcionar un lugar adecuado y personal perfectamente capacitado (4) que permita la atención del paciente con problemas de distintos tipos y debe facilitarse el acceso a la atención adecuada para poder comenzar lo antes posible el tratamiento de los traumatismos y las enfermedades agudas (5).
Se ha reportado en el mundo un aumento importante en el número de pacientes que acuden a los servicios de urgencias médicas, México ha experimentado un incremento porcentual de más del 62\% en solo 3 años (6) en hospitales de Valencia, España, el ingreso a hospitales llega a ser de 300400 pacientes por cada 1000 habitantes al año (7), lo que es motivo de preocupación general, ya que esto conlleva a una demanda que en muchos casos tiende a colmar dichos servicios y atenta con la calidad de los mismos $(8,9)$.

En relación a los acontecimientos de atención de urgencias uno de los principales motivos de consulta en urgencias de instituciones ampliamente reconocidas en la ciudad de Bogotá, para el caso de Colombia como es la Fundación Clínica A. Shaio, centro de referencia para enfermedad coronaria es el dolor torácico. Conforme a datos entregados fueron atendidos 3.756 pacientes que consultaron por dolor torácico; dentro de los cuales 2.525 (67.2\%), fueron dados de alta. De este grupo 309 pacientes (8.22\%) ingresaron con diagnóstico de infarto agudo del miocardio; $552(14.69 \%)$ se hospitalizaron con diagnóstico de angina. $370 \mathrm{pa}-$ cientes con riesgo intermedio definido como pacientes con al menos dos factores de riesgo con dolor típico o atípico y electrocardiograma normal o no diagnóstico $(9.85 \%)$ ingresaron para completar el estudio diagnóstico (10).

Se define la urgencia médica como toda aquella situación que lleva al paciente, al testigo o a sus familiares, a solicitar asistencia médica inmediata. Dicha definición, acorde con los criterios expuestos por las diferentes instituciones internacionales (OMS, Consejo de Europa) pretende integrar en dicho concepto la totalidad de las situaciones vividas por la población como urgencia - médica, diferenciándolas a su vez de otros problemas de carácter no sanitario que por su naturaleza requieren asimismo de respuestas diferenciadas, aunque susceptibles de actuar coordinadamente en caso necesario (11). 
El Departamento de urgencias es una unidad integral que el hospital dedica, para recibir, estabilizar y manejar pacientes que se presentan con una gran variedad de condiciones urgentes ó donde los pacientes se presentan solos o mediante referencia. Los cuidados deben ser con un alto estándar de calidad y donde la comunidad percibe la necesidad de atención aguda o urgente, incluyendo la admisión hospitalaria, para proporcionar esta atención el servicio de urgencias debe ser organizado y administrado por personal que idealmente conozca de las urgencias (urgenciologo) específico de ese servicio, además de contar con conocimientos de administración (12).

La intervención avanzada de la ayuda de la vida (ALCS) es el conjunto de competencias laborales de atención médica prehospitalaria avanzada en el lugar del incidente, más allá del alcance de la práctica de un técnico-básico médico de la emergencia (EMT- Básico). Durante el traslado de la víctima, hasta su entrega al hospital receptor. Esta actividad está enfocada a víctimas con cuadros críticos agudos derivados de padecimientos cardiovasculares, respiratorios, neurológicos, quirúrgicos, ginecobstétricos, traumáticos e urgencias ambientales. Además de la aplicación y uso de líquidos cristaloides. Indicaciones de medicamentos para tratamiento de urgencia administrados por otros medios, ejemplo: inyección intramuscular o subcutánea, suministro de medicamentos vía oral, sublingual o nebulizada como también la atención traumática y cardiaca avanzada para preservar la vida (13).

La urgencia médica se concibe como todo padecimiento de orden agudo o crónico agudizado, que el paciente percibe o siente que amenaza su vida. Casi nunca es grave y puede ser atendido por consulta externa. Las cifras varían entre los países pero su rango va de $80-85 \%$ de todas las urgencias (11). En concordancia a lo anterior se puede también definir como urgencia real, toda condición médica de instalación súbita que pone en riesgo la vida, un órgano o la función, y que requiere de una atención inmediata. La emergencia médica real se entiende como una todo problema médicoquirúrgico agudo, que ponga en peligro la vida, o la pérdida de un órgano o una función y requiera atención inmediata (14).

El término "triage" proviene del francés "to sort" clasificar, este concepto de fue utilizado de manera inicial en las guerras napoleónicas, el método se utilizaba cuando había muchos lesionados y los recursos eran limitados (15), la prioridad es para los pacientes en condiciones más urgentes. Los servicios de urgencias utilizan este método de manera diaria para valorar los recursos de los servicios y la atención de dichos pacientes (16).

También se denomina como el método de selección y clasificación de pacientes basados en sus necesidades terapéuticas y los recursos disponibles para su atención. Este evento también debe ser aplicado en el sitio del accidente en el momento de seleccionar el hospital al cual se trasladará el accidentado. Para este evento existen dos tipos de situaciones de selección en el de múltiples lesionados cuando el número de pacientes y su gravedad de lesión no sobrepasa la capacidad del hospital para proporcionar la capacidad médica necesaria, se atiende primero a los pacientes graves (riesgo a la vida) y aquellos con lesiones múltiples y en accidentes masivos o desastres, cuando el número de pacientes y su gravedad de lesiones sobrepasan la capacidad de los recursos hospitalarios y humanos, se atenderá primero aquellos con mayores posibilidades de sobrevida, que consuman poco tiempo, equipo, material y personal (11).

Administrativamente, y acorde con el con el Manual de Procedimientos e Intervenciones del Plan Obligatorio de Salud, se incluyen actividades de urgencias: evaluación y atención medica; atención médica que requiera sutura; atención médica con cuidado en observación hasta por 24 horas; atención médica con cuidado en hidratación; evaluación, estabilización y remisión del paciente que lo requiera e interconsulta especializada. Estas acciones son indispensables para el manejo de la urge cia, 
sin embargo para que esta se pueda ejecutar es importante tener en consideración la obligatoriedad de la aplicación de triage por personal idóneo. La realización de este (clasificación de la prioridad para la atención de urgencias) o la toma de decisiones acerca de si un paciente debe recibir o no atención de urgencia en una Institución Prestadora de Servicios de Salud, dicha responsabilidad debe estar a cargo de un profesional de salud debidamente entrenado para tal efecto el paciente calificado como apto para la atención de urgencia no debe exigírsele que su ente previsor de salud tenga contrato o autorización previa para la atención inicial de urgencias.

En consecuencia, las IPS están obligadas a prestar el servicio y las EPS a realizar el pago correspondiente. No debe exigírsele pago previo o copago para la atención inicial de urgencias. En ningún caso la atención inicial de urgencias puede estar condicionada al pago de suma de dinero alguna por parte del usuario del servicio, los periodos de carencia no aplican en casos de atención inicial de urgencias. De conformidad con lo establecido por la ley (17) para la atención inicial de urgencias no puede exigirse el cumplimiento de periodos mínimos de cotización. En consecuencia, el acceso a servicios tales como la internación en Unidad de Cuidados Intensivos o la realización de procedimientos quirúrgicos que se requieran de manera inmediata para estabilizar un paciente en estado crítico, deben ser considerados como parte de la atención inicial de urgencias y por tanto no están sujetos a periodos mínimos de cotización y sus co tos deberán ser reconocidos por la respectiva EPS (18).

La IPS que atienda la urgencia puede adoptar las siguientes conductas alternativas dependiendo la condición del paciente, y si este se encuentra asegurado o está afiliado a una Empresa promotora de salud contributiva o subsidiada se puede continuar con la atención al paciente si obtiene la autorización de la aseguradora (EPS, EPSS, cajas de previsión, entidad de medicina prepagada, etc.) a la cual esté afiliado, o remitirlo a la institución que señale dicha aseguradora, si paciente no está asegu- rado, pero tiene capacidad se debe continuar con el tratamiento siguiente, si éste autoriza, o trasladarlo a la entidad que él o su familia determinen. En el caso de atender un paciente pobre y vulnerable que no esté asegurado es recomendable continuar con la atención al paciente. Cuando la urgencia sea atendida por una entidad pública o por una privada que tenga contrato con el Estado. Si es privada y no tiene contrato con el Estado, debe remitir el paciente a una institución pública (17).

En el caso de las víctimas de accidentes de tránsito, la atención del paciente deberá ser integral, y la remisión a otra institución sólo podrá hacerse si la entidad no cuenta con la capacidad o los recursos para la complejidad del caso para este caso, la ley 100 de 1993 y sus normas reglamentarias, la atención de urgencias debe ser prestada en forma obligatoria a todas las personas, independientemente de la capacidad de pago, por todas las entidades públicas y privadas que presten servicios de salud, este tipo de atención no requiere convenios ni contratos con las entidades aseguradoras, como tampoco autorización ni orden previa por parte de las mismas si se requiere realizar procedimientos adicionales, luego de que se haya estabilizado al paciente y se defina su destino inmediato, es por esto que la atención en urgencias debe ser prestada obligatoriamente por todas las IPS públicas y privadas. Si son atendidas por IPS privadas, éstas le brindarán la atención inicial de urgencias hasta estabilizarlas, y posteriormente deberán remitirlas a una IPS pública, la cual está en la obligación de atenderlas, queda entendido que para ser atendido en urgencias no se requiere copagos ni cuotas moderadoras ni mucho menos debe ser exigido por el prestador. Sin embargo, cuando se acuda a este servicio sin que sea por una verdadera urgencia, el paciente deberá pagar el valor total de la atención. En todo caso, es el médico quien define esta condición (19).

Cuando se trate de atención por enfermedad general el costo de la atención de urgencias es asumido por la respectiva entidad aseguradora, pero si la urgencia es generada por acciones terroristas, 
catástrofes naturales o accidentes de tránsito ocasionados por vehículos con pólizas vencidas o falsas, los gastos son asumidos por el fondo de solidaridad y garantía, FOSYGA, quien paga directamente a la institución que haya prestado el servicio. Las IPS tienen la responsabilidad de Identificar la causa de la urgencia a fin de contar con los soportes necesarios para los reembolsos a que haya lugar, cuando se trate accidente de trabajo o enfermedad profesional, accidente de tránsito, acciones terroristas o catástrofes naturales, como también identificar si es afiliado a algún sistema de aseguramiento, o si se trata de una persona pobre no asegurada, así como el municipio y departamento habitual de residencia, a fin de conocer la entidad responsable del pago de los servicios prestados (17).

Es primordial calificar en primera instancia el origen del accidente de trabajo o enfermedad laboral del paciente, e informar a la EPS a la cual se encuentre afiliado, dentro de los dos días hábiles siguientes a la ocurrencia del accidente de trabajo o a la primera calificación de la enfermedad profesional, para Informar a la respectiva EPS el ingreso de su afiliado, dentro de las doce (12) horas hábiles siguientes a la solicitud del servicio, so pena del no pago respectivo. Se exceptúan los casos en que por fuerza mayor no se pueda informar oportunamente. De lo contrario se debe Informar, dentro de las 12 horas hábiles siguientes a la solicitud del servicio, a la respectiva Dirección de Salud el ingreso de pacientes pobres no asegurados, y de pacientes con capacidad de pago que no se encuentren afiliados al Sistema General de Seguridad Social en Salud (20); la no atención en urgencias o las irregularidades en este tipo de atención pueden derivarse en denuncias en primera instancia ante la dirección de salud del departamento, distrito o municipio que corresponda y posteriormente ante la Superintendencia Nacional de Salud, entidad que adelantará la respectiva investigación y podrá aplicar las sanciones, en caso de que sea necesario $(21,22)$.

En Medio Oriente, específicamente Israel y Pakistán, tienen las más modernas redes de urgencias del hemisferio oriental, sin embargo en un estudio experimental cuantitativo con una muestra de 22 zonas rurales y 20 centros de salud urbanos en los distritos Faisalabad y Peshawar en Pakistán se evaluaron a los proveedores del servicio de urgencia (IPS), obteniendo de este una muestra de 44 instituciones prestadoras de servicios de salud donde el $98 \%$ no estaban satisfechos con la atención de emergencia. Y la mayoría de sus participantes 36/44 (82\%) mencionaron que la atención intramural y las ambulancias no funcionan correctamente en el sistema de gobierno como también la mayoría de los proveedores de atención sanitaria 43/44 (98\%) merecen la opinión que sus instalaciones no están suficientemente equipadas para tratar emergencias (23). Como también se sugirió que la evaluación de la atención de urgencia debería ser parte del análisis de los sistemas de salud en Pakistán, debido a que las múltiples deficiencias en la atención de emergencia a nivel de distrito en el Pakistán deben dar prioridad a la atención de emergencia que responda a las necesidades de los pacientes orientando esfuerzos en dotar a la atención de emergencia en los distritos que instalaciones y organizar una red de ambulancias eficiente, concordante con lo anterior la atención en los países en desarrollo no se ha centrado tradicionalmente en la atención médica de emergencia (24).

Paradójicamente, es este tipo de atención el que mayor prioridad requiere estos países, donde las tasas de lesiones son más altas y menos actividades de control se han llevado a cabo. La atención de emergencia puede hacer una importante contribución a la reducción de muertes evitables y de discapacidad, especialmente en los países de bajos y medianos ingresos (25). Sin embargo, el estado de la atención de la salud en Pakistán indica que el sistema de salud pública ha empezado a enfatizar más en la atención médica de emergencia. Los datos procedentes de siete países en desarrollo muestran que 14 de los 21 hospitales carecen de un adecuado sistema de triage y el método para la evaluación inicial del paciente es demorado en el tratamiento (26). De acuerdo a esto la atención en países de medio 
oriente no dista de la atención que Colombia puede ofrecer a su población, solo que al incrementarse la demanda por motivos de urgencia en virtud del orden público (atentados, combates y bombardeos) ponen en continuo a la red hospitalaria de urgencia en acción sobresaliendo en estas regiones, aunque esto no podría indicar que el sistema de salud de estos sea superior en desempeño o efectividad clínica ante una urgencia vital.

El uso de los servicios hospitalarios de emergencia (EHS) se ha incrementado de manera constante en España en la última década, motivado esto por la creciente llegada de inmigrantes y turistas que acuden a este servicio. Los inmigrantes utilizan más el servicio urgencia que los espańoles nacidos en la población. Hay diferencias en el uso de la urgencia por zona de origen. Las mujeres inmigrantes de los países de bajos ingresos tienen el doble de probabilidades de utilizar el servicio de urgencia que el español promedio, los inmigrantes hombres utilizan los servicios de urgencias $42 \%$ más que los varones nacionales. Las diferencias en los patrones de utilización son especialmente identificadas para los hombres y las mujeres inmigrantes habitantes en el pais.

Las razones más comunes para las visitas al hospital y la admisión de los inmigrantes se relacionan con las necesidades de salud de la población más joven (27); los motivos frecuentes fueron obstétrica- ginecológica y tratamiento de la medicina general.

$\mathrm{Al}$ analizar los diagnósticos más frecuentes por grupos de edad, hemos observado que los inmigrantes y los nacidos en los grupos son similares. Es posible, sin embargo, que pueden existir entre los diagnósticos problemas de salud relacionados con enfermedades tropicales u otras afecciones endémicas entre los inmigrantes zona que pueden representar un importante impacto sobre la salud pública de la zona (28). Con referencia a los ingresos hospitalarios, hay un patrón diferente en los hombres y las mujeres. Si bien los inmigrantes de sexo masculino tuvieron un riesgo significativamente inferior de in- greso en el hospital que los espańoles promedio, las mujeres inmigrantes fueron admitidas con más frecuencia que los españoles. Con respecto a las consultas ginecológica y obstétrica el riesgo de ingreso hospitalario, fue similar tanto para inmigrantes y españoles (29). Esta problemática aplicada a la realidad colombiana se asimila al paciente vinculado que no tiene afiliación al sistema y que debe ser tratado por la institución en salud como lo indica el decreto 806 de 1998, mencionado en este mismo texto.

\section{Materiales y métodos}

Estudio descriptivo consistente en una búsqueda documental y estadística en fuente variadas de información entre las cuales se destacan libros, artículos e informes relacionados con el servicio de salud y páginas web con datos y conceptos de diferentes autores y organizaciones vinculadas con la atención de urgencias y atención sanitaria, tomando como principal insumo los estudios relevantes de la encuesta nacional de salud y la observación de los servicios hospitalarios de emergencia (EHS) en Medio Oriente, Europa y España.

Esta investigación cobra especial interés debido a la inexistencia de estudios que revelen el comportamiento de los servicios de salud en el país y específicamente en la ciudad de Cartagena, donde a falta de datos que identifiquen la condición de estos servicios, se requiere de una revisión estadística del servicio de urgencia, justificación esencial de esta iniciativa de investigación que utilizó tablas y análisis invariadoque estudian el fenómeno en cuestión para dar respuesta a los interrogantes sobre características de la atención de urgencia en la ciudad de Cartagena, Colombia.

\section{Resultados}

Distinto a los datos de países de Oriente Medio donde el grado de satisfacción en el servicio de urgencias es bajo y en España donde la variación entre el inmigrante y el nacional es marcada debido 
a la aparición del inmigrante africano y americano que han desencadenado un verdadera problemática de salud pública en el país ibérico; en Colombia aún no se han discriminado estadísticas relevantes sobre este particular para lo cual se recurrió a la encuesta nacional de salud realizada en el año 2007, publicada en el mes de abril, módulo 2 aplicativo para hospitalización y urgencias, ítem VII, numerales 701 a 720, (19 ítems, válidos para la valoración de escalas, evitando sobre valorar el instrumento o desestimarlo.) No sin antes confrontar las estadísticas difundidas para la actualización de la realidad en salud colombiana, más directamente el servicio de urgencias, de los encuestados 996566 son mujeres (61\%); mientras que 637149 son hombres (39\%).

Esta encuesta debe renovar datos cada 5 ańos y publicarse en 2015; sin embargo esta información se encuentra en proceso de recolección por parte del Ministerio de Salud, por tal razón se remite al estudio del año 2007condicion decepcionante al no disponer de referentes de mayor actualidad a primer semestre de 2015. Ahora bien, el panorama del departamento de Bolívar, más exactamente Cartagena no está totalmente definida en cuanto a este servicio del que se ha hablado mucho pero no se ha puntualizado, por lo cual se procede a dar una vista objetiva de la atención de urgencias entre el resto del país y la ciudad de Cartagena.

Tabla 1. Clasificación de las urgencias en Colombia.

\begin{tabular}{|r|r|r|}
\hline Servicio & Cantidad & Porcentaje \\
\hline $\begin{array}{r}\text { Urgencias no } \\
\text { obstétrica }\end{array}$ & 1537252 & $94,0 \%$ \\
\hline $\begin{array}{r}\text { Urgencias } \\
\text { obstétricas }\end{array}$ & 96463 & $6 \%$ \\
\hline TOTAL & 1633715 & $100 \%$ \\
\hline
\end{tabular}

Fuente. Encuesta nacional de salud, 2007.

En la tabla 1, se encuentra que la mayoría de las consultas encuestadas pertenecen a urgencias no obstétricas, mientras el 6\% de estas tienen relación al parto, entendidas estas últimas como una pri ridad institucional para con las parturientas y por tal razón son atendidas con preferente disposición. Mientras en Cartagena el 86\% de las consultas encuestadas pertenecen a urgencias no obstétricas, y el $14 \%$ de estas tienen relación al parto. De los encuestados 915 son mujeres (55\%); mientras que 735 son hombres (45\%)

Tabla 2. Motivos de la consulta de urgencias en Colombia.

\begin{tabular}{|r|r|r|}
\hline $\begin{array}{r}\text { Motivo de la } \\
\text { urgencia }\end{array}$ & Cantidad & Porcentaje \\
\hline $\begin{array}{r}\text { Enfermedad } \\
\text { física larga } \\
\text { duración }\end{array}$ & 206744 & $13 \%$ \\
\hline $\begin{array}{r}\text { Enfermedad } \\
\text { física malestar } \\
\text { reciente }\end{array}$ & 1003719 & $61 \%$ \\
\hline Problema mental & 4847 & $0,2 \%$ \\
\hline $\begin{array}{r}\text { Disminución } \\
\text { capacidad física }\end{array}$ & 6421 & $0,39 \%$ \\
\hline Accidente & 275322 & $17 \%$ \\
\hline Violencia & 17232 & $1,05 \%$ \\
\hline Parto & 22161 & $1,35 \%$ \\
\hline Otros & 97207 & $6 \%$ \\
\hline TOTAL & 1633653 & $100 \%$ \\
\hline
\end{tabular}

Fuente. Encuesta nacional de salud, 2007.

La Tabla 2 referente a motivos de urgencias revela que el $61 \%$ de las asistencias al servicio de urgencias en Colombia corresponden a padecimientos agudos o de reciente aparición, mientras otro importante $13 \%$ está relacionado con malestares físicos de larga duración, aunque se podría inferir que los padecimientos agudos tiene mayor frecuencia pero menor estancia en los recintos hospitalarios. En cuanto a esta situación Cartagena con un 37\% de las asistencias al servicio de urge cias tienen relación a padecimientos agudos o de reciente aparición, el $18 \%$ se relaciona con accidentes mayormente de tránsito, seguido en importancia por el parto $(14 \%)$ y la violencia (13\%).

Tabla 3. ¿Pagó o va a pagar copago por la atención en urgencias?

\begin{tabular}{|r|r|}
\hline SÍ & $26 \%$ \\
\hline NO & $\mathbf{7 4 \%}$ \\
\hline TOTAL & $\mathbf{1 0 0} \%$ \\
\hline
\end{tabular}

Fuente. Encuesta nacional de salud, 2007. 
En la Tabla 3 se resume como una minoría de los pacientes encuestados aún cree que la atención de urgencias debe pagarse, mientras un mayor porcentaje está informado que por este servicio no se cobra cuota moderadora ni copago. Como objeto de revisión debe indicarse que a diferencia de la antes expuesto, la urgencia no vital pude ser objeto de cobro por parte de la institución prestadora de servicios de salud tal como lo indica el acuerdo 260 de febrero 4 de 2004. El 12\% de los pacientes encuestados en Cartagena desconocen que en la atención de urgencias no se paga, mientras el 88\% sabe que por este servicio no se cobra valor alguno.

Tabla 4. ¿Pagó o va a pagar por los materiales para la atención en urgencias?

\begin{tabular}{|r|r|}
\hline SÍ & $0,5 \%$ \\
\hline NO & $95,5 \%$ \\
\hline TOTAL & $\mathbf{1 0 0} \%$ \\
\hline
\end{tabular}

Fuente. Encuesta nacional de salud, 2007.

Paradójicamente a lo que sucede en muchas IPS del país en la Tabla 4 se encuentra que casi la totalidad de los pacientes afirma que no gastó dinero alguno en la compra de materiales o para su atención; caso contrario con la papelería, que produjo gastos a cargo de los pacientes y/o acompañantes en un $44 \%$ de los consultados. En un número significativo de IPS de Cartagena (55\%) los pacientes afirman que compraron materiales para su atención; otro $44 \%$ de los consultados en Cartagena afirman no haber gastado dinero por este concepto.

Tabla 5. ¿Sintió molestias por las condiciones físicas de la sala de espera en urgencias?

\begin{tabular}{|r|r|}
\hline SÍ & $11 \%$ \\
\hline NO & $89 \%$ \\
\hline TOTAL & $\mathbf{1 0 0} \%$ \\
\hline
\end{tabular}

Fuente. Encuesta nacional de salud, 2007.

Como lo refleja la Tabla 5 un porcentaje de los pacientes afirmó sentirse incomodo en algún momento de su espera en la sala de urgencias, debido a los tiempos de atención y la infraestructura logística de la sala de urgencias, la mayoría notificó estar conforme. El 31\% de los pacientes afirmó sentirse incomodo en algún momento de su espera en la sala de urgencias en las IPS de Cartagena, ya por estar de pie un prolongado tiempo y por las incomodas instalaciones.

Tabla 6. ¿¿Sintió molestias por la forma en que lo atendieron?

\begin{tabular}{|r|r|}
\hline SÍ & $3 \%$ \\
\hline NO & $97 \%$ \\
\hline TOTAL & $\mathbf{1 0 0 \%}$ \\
\hline
\end{tabular}

Fuente. Encuesta nacional de salud, 2007.

En la Tabla 6 muy pocos pacientes afirmaron sentirse incomodo en algún momento por la forma en que lo atendieron o hablaron las personas que lo atendieron en el servicio de urgencias. Por el anterior análisis se puede afirmar que son más las falencias de tipo administrativo en los sitios de atención de urgencias, que las debilidades de la asistencia propiamente dicha, por lo cual el administrador de los servicios de salud debe afrontar un verdadero reto para acoplar la estructura administrativa de su IPS la demanda de los servicios de salud en especial la atención de urgencia, que es un espacio en estado crítico en las IPS en Colombia. Paralelamente en la ciudad de Cartagena el $7 \%$ de los pacientes afirmó sentirse incomodo en algún momento por la forma en que lo atendieron o hablaron las personas que lo atendieron el servicio de urgencias.

\section{Discusión}

En Cartagena no existe la infraestructura suficiente para atender una emergencia de gran magnitud. En condiciones normales, la red de urgencias no puede atender la cantidad de pacientes que llegan a solicitar ese servicio, descartando el caso de una verdadera emergencia colectiva. Actualmente en Cartagena hay un déficit de 380 camas. Pues sólo existen 1.057 camas (esto sumando la red pública y privada) lo que equivale a que hay un promedio de 1,05 camas para cada mil habitantes, una de las más bajas del país. Mientras el decreto 412 de 1992 exige que se cuente con los recursos ade- 
cuados y la dotación para la atención urgencias, acorde con los niveles y grados de complejidad (17) para Bogotá, hay un promedio de 1,45 por cada mil habitantes, en Medellín aumenta a 2,05 , también por cada mil habitantes, de acuerdo con las estadísticas de la Asociación Médica Sindical de Colombia (ASMEDAS), mientras Cartagena adolece de recursos para dotar su red prestadora servicios de urgencia pues a diario, unas 80 ó 90 personas tienen que utilizar las camillas, las sillas o el piso, a la espera de una atención de urgencia (12).

Al contrario de lo determinado por la EMT-Basic, donde la atención debe realizarse bajo condiciones de confort y asepsia, la mayoría de la urgencias médicas de Cartagena corresponden a cuadros agudos y accidentes, para lo cual se debe disponer de una infraestructura adecuada pero la realidad indica de forma clara que la red de urgencias es precaria en esta ciudad, administrativamente se deben gestionar más recursos para la consecución de espacios y aéreas habilitadas para este tipo de servicios, ya que en Cartagena existen muchas IPS de atención ambulatoria, pero pocas urgencias (17).

El Hospital Universitario del Caribe HUC, el centro asistencial de mayor demanda en los estratos bajos de la ciudad, y en la actualidad solamente ofrece servicios en 4 de los 10 pisos de su edificación, luego de su reapertura en julio del año 2006, tras tres años de cierre de las 289 camas que ofrecía cuando inició labores, el HUC sólo cuenta con 137 camas disponibles, y aun así, sigue siendo el que recibe el mayor número de personas de extracción popular y el sistema de atención de emergencias del distrito es muy deficiente, lo que hace que la salud quede en manos de la red privada, por eso se necesita fortalecer cuanto antes la red de urgencias, para que entre otros, no se cometan atropellos ni se vulnere el derecho a ser atendido en la urgencia por no contar con una afiliación a un ente previsor como lo indica el decreto 806 en el sistema de salud, pero ante el desconocimiento del usuario en cuanto al servicio de urgencia en Cartagena.

También ha perjudicado a esta importante sección del ente prestador: urgencias que realmente no lo son, dificultades para informar y reubicar al enfermo, pacientes que no asisten a la urgencia por creer que este servicio les será cobrado directamente a ellos, agravando su actual estado de salud e incluso pacientes que asisten a este servicio creyendo que su atención tiene un costo que deberán pagar, atenciones en la urgencia que son realizadas de forma deficiente, materiales que se han agotado y deben ser conseguidos en la farmacia con dineros del usuario o acompañante de este en la urgencia dejan mucho que desear de la actual atención de urgencia que reciben los cartageneros $(12,30)$.

Los resultados de esta investigación, así como los análisis que se generan de los mismos, permiten concluir que: asistir a una atención de urgencia ya sea de red pública o privada en Cartagena es una decisión bastante incómoda, como lo refiriere el resultado de la encuesta de salud aplicada, las instalaciones destinadas a este tipo de atención en la mayoría de las IPS que ofrecen este servicio son deficientes, incomodas, existe hacimiento en las áreas de espera, consultorios, pasillos y cubículos para pacientes. En este caso el administrador de los servicios de salud debe contemplar un plan de contingencia útil para resolver situaciones donde la prioridad sea la comodidad y rapidez en la atención, pues son estos los motivos que más afectaron la opinión de los encuestados en esta ciudad.

De esta forma, es importante considerar el administrador de los servicios de salud, quien implementará estrategias para la maximización de los recursos del ente prestador de servicios de salud, más puntualmente, la urgencia (31-33). Depurando los procesos y las mejoras de infraestructura, conformando un adecuado sistema de triage y referencia interinstitucional, que sea efectivo en 
su normal que hacer y eventos en que la demanda del servicio de urgencia de incremente.

Se debe tener presente que la mayoría de las quejas en la urgencia tuvieron relación con la logística y trámites administrativos al interior de esta, por lo cual son estos aspectos lo que se deben mejorar y priorizar a la hora de la resolución y planeación administrativa, por esto el administrador de los servicios de salud debe liderar una gestión orientada a la mejora de las condiciones de infraestructura, dotación, suministro médico y administrativo, información y atención al cliente externo, que necesita ser atendido con calidad y respeto por su integridad. De la misma forma, se requiere depurar en su plan de mejoramiento continuo los procesos de referencia, facturación de urgencias y resolución de casos para así aportar materiales e insumos importantes que mejoren el desempeño de la Institución en un área tan crítica e importante como lo es la urgencia.

\section{Agradecimientos}

Por su apoyo y colaboración al ingeniero Eduardo Mesías Salamanca Blanco y al Dr. Ricardo Vivas Reyes, Grupo CIPTEC de la Facultad de Ingeniería, Fundación Universitaria Tecnológico Comfenalco.

\section{Referencias}

1. Ministerio de Salud Pública. Establece las actividades, procedimientos e intervenciones de demanda inducida y obligatorio cumplimiento y se adoptan las normas técnicas. Santa Fe de Bogotá: Diario Oficial 40368; 1992 p.35.

2. Sánchez Padilla L. www.portalesmedicos.com. [Internet]. Portales médicos. 2015 [cited 26 April 2008]. Available from: http://www.portalesmedicos.com/

3. Wilkins E. Medicina de Urgencia. La Habana: Edición revolucionaria; 1984 p 4-11.

4. Hamilton R, Hayes B, Briggs. Mantenimiento de la vida. Medicina de urgencia. La Habana: Edición Revolucionaria; 1984 p. $18-51$.

5. Sanders C. Clínica Medicina de urgencia. La Habana: Edición Revolucionaria; 1984 p 71-408.
6. García Farinas A, Rodríguez Salvá A, De vos P, Jova Morel R, Bonet Gorbea M, Garcio Roche R et al. Costos del subsistema de urgencias en la atención primaria de salud en Cuba. Revista Cubana Salud pública. 2006; 32(1):1-6.

7. Pereda Rodríguez J, Díaz Sánchez I, Pereda Rodríguez R, Sosa Acosta A. Filtro sanitario en las urgencias médicas. Un problema a reajustar. Revista Cubana Salud Publica 2001; 40 (3):181-188

8. Pereda Rodríguez, J., Díaz Sánchez, I., \& Pereda Rodríguez, R. Urgencias clínicas. Revista Cubana medicina 2001; 23(4) 329-335.

9. Fajardo-Ortiz G., Ramirez-Fernandez FA. Utilización del servicio de urgencias en un hospital de especialidades. Cir \& Cir 2000; 68:164-8.

10. Merlano Sonia, Murgueitio Ramón, Rodríguez Edgard. La perfusión miocárdica. Imagen validada y costo-efectiva: Punto de vista desde el análisis crítico. Rev. Col. Cardiol. [Internet]. 2008 Dec [cited 2017 Mar 23] ; 15( 6 ): 247-254. Available from: http://www.scielo.org.co/scielo.php?script=sci_arttext\&pid=S0120-56332008000600001\&lng=en.

11. Villatoro MA., Carrasco NM., Sánchez GM., Uso inapropiado de la terapia antisecretora gástrica en Urgencias. Arch Med Urg Mex 2012; 4 (1) 6-12.

12. Carpio Coloma, A., Hidalgo Murillo, A., Lago Díaz, N., Muńoz Kaltzakorta, G., Onrubia Calvo, S., \& Periáñez Serna, I. Efectividad de la implantación de una consulta de enfermería en Urgencias. Enfermería de Cantabria [Revista en línea], 2011 Febrero - Marzo [Acceso 02-05-2015] 1(5) Disponible en http://www.enfermeriacantabria.com/enfermeriacantabria/ web/articulos/2/14.

13. Redices [base de datos en Internet]. Bravo Corrales, J. D., Flórez Lemos, D. P., \& Salazar Correa, E. M: Servicios de urgencias en Colombia, el caso Medellín; [base de datos en Internet];Medellin,Bibliteca Fundadores; 2013 [fecha de acceso en marzo 9 de de 2015]. Disponible en: http://bdigital.ces.edu. co:8080/repositorio/handle/10946/828.

14. Quintana Miró., R. Coordinación de urgencias. Avances en emergencias y resucitación, $3^{a}$ ed.Barcelona:1998; 37-43.

15. strada, E. Triage Sistem. Nurs Clin North Am, 1981; 16 (1):13-24.

16. Soler W., Gómez Muñoz M., Bragulat E., Álvarez A.. El triaje: herramienta fundamental en urgencias y emergencias. Anales Sis San Navarra [Internet]. 2010 [citado 2017 Mar 23] ; 33( Suppl 1 ): 55-68. Disponible en: http://scielo.isciii.es/scielo. php?script=sci_arttext $\&$ pid=S1137-66272010000200008\&l$\mathrm{ng}=\mathrm{es}$.

17. Ministerio de Salud Pública. Afiliación al sistema general de seguridad social. Santa Fe de Bogotá, Cundinamarca,Colombia: Diario Oficial 43291;1998 p 3.

18. Ministerio de Salud. Instrucciones sobre la atención oportuna a la población, especialmente cuando está en peligro la vida de los pacientes. Santa Fe de Bogotá, Cundinamarca, Colombia: Diario Oficial; 2006 p 1. 
19. Presidencia de la república. Por el cual se modifican los Decretos 1486 de 1994, 1922 de 1994, 723 de 1997, y 046 y 047 y se dictan otras disposiciones. Santa Fe de Bogotá, Cundinamarca, Colombia: Diario Oficial; 2000 p 5.

20. Asamblea Nacional Constituyente. Constitución Política de Colombia. Constitución. Santa Fe de Bogotá, Cundinamarca, Colombia: Diario Oficial; 1991 p 4.

21. Congreso de la Republica de Colombia. Ley 100. Sistema General de seguridad Social. Santa Fe de Bogotá, Cundinamarca, Colombia: Diario Oficial; 1993 p 12.

22. Ministerio de hacienda y crédito público. Por el cual se regula integralmente el seguro obligatorio de daños corporales causados a las personas en accidentes de tránsito. Decreto 1032. Santa fe de Bogotá, Cundinamarca, Colombia: Diario Oficial 39.802; 1991 p 10.

23. Razzak JA, Hyder AA, Akhtar T, Khan M, Khan UR. Assessing emergency medical care in low income countries: a pilot study from Pakistan. BMC Emerg Med. 2008 Jul 3;8:8. Published online 2008 July 3. doi: 10.1186/1471-227X-8-8.

24. Mock C, Quansah R, Krishnan R, Arreola-Risa C, Rivara F. Strengthening the prevention and care of injuries worldwide. Lancet. 2004; 363:2172-2179.

25. Kobusingye, OC, Hyder, AA, Bishai, D, Hicks, ER, Mock, $\mathrm{C}$, and Joshipura, M. Emergency medical systems in low- and middle- income countries: recommendations for action. Bull World Health Organ. 2005; 83: 626-631.

26. Nolan T, Angos P, Cunha AJ, Muhe L, Qazi S, Simoes EA, Tamburlini G, Weber M, Pierce NF. Quality of hospital care for seriously ill children in less-developed countries. Lancet. 2001; Jan 13;357(9250):106-110.

27. Berra S, Elorza Rocard JM, Bartomeu N, Hausmann S, Serra- Sutton V, Rajmil L. Necessitats en salud i utilització dels serveis sanitaris en la població immigrant a Catalunya; Revisió exhaustiva de la literatura científica. Barcelona: Agència dAvaluació de Tecnología i Recerca Mèdiques; 2004; Anales de medicina; $\mathrm{p} 87$.

28. Roca, C., \& de Balanzo, X.. Imported diseases in immigrants: myth and reality. An Sist Sanit Navar, 2006; 139-144.

29. Cacciani, L., Baglio, G., Rossi, L., Materia, E., Marceca, M., Geraci, S., y otros. (2006). Hospitalisation among immigrants in Italy. Emerg Themes Epidemiology, 2006; 3:4. doi: 10.1186/1742-7622-3-4].

30. Cortés M, Posada J, Duque P, Garzón A. Erradicación de la pobreza y calidad en la salud: Elementos indispensables para alcanzar la cohesión social en Colombia. NOVA. 2013, 11 (20): 97 - 119.

31. Cortés G, Campuzano S, Turriago A. La evasión y elusión en el sistema general de riesgos profesionales de las organizaciones afiliadas al sistema general de seguridad social en la ciudad de Bogotá, DC. 2000 - 2006. NOVA. 2011; 9 (16): 138 - 148.
32. Millan, J. and L. Yunda. An Open-Access Web-based medical image atlas for collaborative Medical image sharing, processing, Web Semantic searching and analysis with uses in medical training, research and second opinion of cases. 2014; Nova 12(22): 143-150.

33. Flórez, R. A. N. Avances y perspectivas en Síndrome de Asperger. 2014; Nova, 12(21). 